\title{
The use of new anticoagulant drugs in ventricular assist devices: Another brick in the wall?
}

\author{
Antonio Miceli, MD, PhD, and Mattia Glauber, MD
}

\author{
From the Centro Cardiotoracico, Istituto Clinico Sant'Ambrogio, Gruppo Ospedaliero San Donato, Milan, Italy. \\ Disclosures: Authors have nothing to disclose with regard to commercial support. \\ Received for publication Jan 1, 2016; accepted for publication Jan 5, 2016; available ahead of print Feb 2, 2016. \\ Address for reprints: Antonio Miceli, MD, PhD, Istituto Clinico Sant'Ambrogio, Via Faravelli 16, 20149 Milano, \\ Italy (E-mail: antoniomiceli79@alice.it). \\ J Thorac Cardiovasc Surg 2016;151:e83-4 \\ $0022-5223 / \$ 36.00$ \\ Copyright (c) 2016 by The American Association for Thoracic Surgery \\ http://dx.doi.org/10.1016/j.jtcvs.2016.01.008
}

The uses of mechanical circulatory assist devices for patients with end-stage heart failure waiting for cardiac transplant or as destination therapy have increased dramatically in recent years. ${ }^{1}$ From 2007 to 2013, there was a 14-fold increase in the number of left ventricular assistance devices implanted, and this rate is expected to increase. Although patient outcomes have improved with the advancement of device technology, bleeding still remains the most frequent and feared complication related to the use of these devices. The incidence of gastrointestinal bleeding in patients with continuous-flow left ventricular assist devices is $20 \%$, whereas the rate of gastrointestinal bleeding recurrence is about $35 \%$.

Angiodysplasia, impaired platelet function, and acquired von Willebrand disease caused by the nonphysiologic continuous flow of the mechanical support have all been proposed as possible mechanisms responsible for bleeding. ${ }^{3}$ The strict observation of antithrombotic treatment plays a crucial role in determining this complication. Although a life-threatening bleeding event necessitates immediate cessation of anticoagulant and antiplatelet drugs, along with the need for warfarin reversal and platelet transfusion, a patient with mild to moderate bleeding requires an individualized assessment to identify the best strategy to minimize bleeding while maintaining anticoagulant treatment and thus avoiding thrombotic events at a later time. ${ }^{4}$ In this setting, the use of new oral anticoagulants might be a reasonable alternative to warfarin treatment.

In this issue of the Journal, Pollari and colleagues ${ }^{5}$ have reported their experience with a new anticoagulant drug, apixaban, in the management of a single patient with a Heart Mate II device (Thoratec Corporation, Pleasanton, Calif) who had multiple gastrointestinal bleeding episodes as a result of warfarin intolerance. Interestingly, after 1 year neither gastrointestinal bleeding episodes nor thrombotic events occurred. The case report presented by Pollari and colleagues ${ }^{5}$ outlines a significant problem that physicians often face with mechanical circulatory support, that of weighing the risks of thrombosis when lowering or stopping anticoagulation therapy against the risks of bleeding

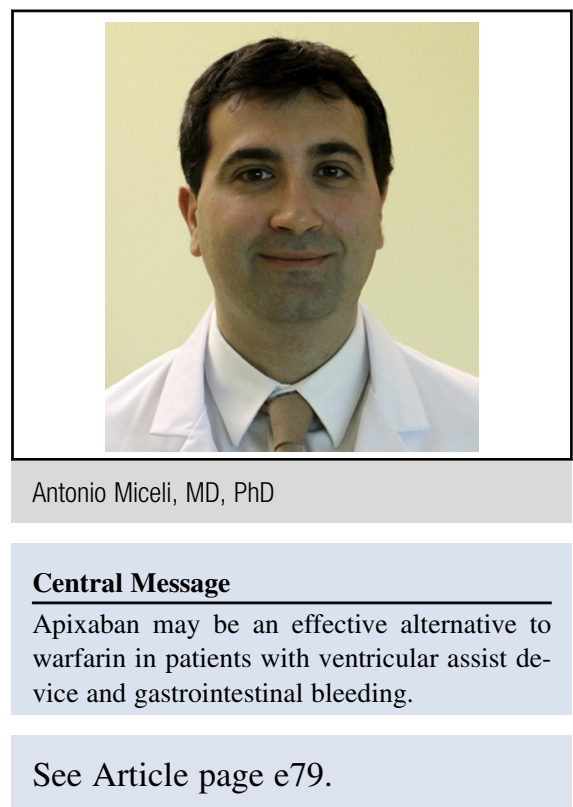

during anticoagulation therapy. Several centers advise withholding any antithrombotic treatment after several gastrointestinal bleeding episodes. . $^{3,7}$ It has been shown that the management of a left ventricular assist device without anticoagulation after major bleeding is possible, although the studies demonstrating this are relatively small, and no long-term follow-up has been reported. ${ }^{8}$ Nevertheless, Stulak and associates ${ }^{9}$ found that patients with a previous gastrointestinal bleeding episode were 7-fold more likely to have a thromboembolic event, suggesting that stopping anticoagulation may not be appropriate.

Conversely, even though a multidisciplinary and ethical discussion was required, this case report represents an important opening up of alternative strategies for anticoagulation protocols. Novel anticoagulant drugs, including dabigatran, apixaban, rivaroxaban, and edoxaban, have been developed as alternative to warfarin for the treatment of atrial fibrillation and for primary venous thromboembolism prevention. These drugs are non-vitamin $\mathrm{K}$ antagonist anticoagulants and act as direct thrombin inhibitors (dabigatran) or factor Xa inhibitors (apixaban, rivaroxaban, and edoxaban). ${ }^{10}$ A meta-analysis of more than 44,000 patients with atrial fibrillation enrolled in randomized, controlled trials has concluded that new oral anticoagulants carry a decreased risk for all cause stroke and systemic embolism, ischemic and hemorrhagic stroke, all-cause mortality and vascular mortality. ${ }^{11}$ In addition, random assignment to 
receive a new oral anticoagulant was associated with a lower risk of intracranial bleeding.

Despite these promising results, some words of caution are required. First, dabigatran has been found to be inferior to warfarin for preventing thromboembolic events in patients with mechanical heart valves. ${ }^{12}$ Second, the major randomized. controlled trials on dabigatran and rivaroxaban have reported an increased risk of gastrointestinal bleeding, whereas apixaban has shown to be not superior to warfarin, despite a trend toward a lower gastrointestinal bleeding rate. ${ }^{13}$ Finally, excluding dabigatran, the effect of which is reversed by idrazumab, these new anticoagulants do not have any reversing agents. These points raise some concerns as to their safety in the field of mechanical circulatory support.

In conclusion, Pollari and colleagues ${ }^{5}$ have helped to open up new alternative strategies for anticoagulant protocols in patients with ventricular assist devices. Apixaban might be a valid alternative to warfarin for this setting of patients; however, more data are required to confirm this hypothesis.

\section{References}

1. Kirklin JK, Naftel DC, Pagani FD, Kormos RL, Stevenson LW, Blume ED, et al. Sixth INTERMATCS annual report: a 10,000-patient database. J Heart Lung Transplant. 2014;33:555-64. Erratum in: J Heart Lung Transplant. 2015;34: 1356.

2. Crow S, John R, Boyle A, Shumway S, Liao K, Colvin-Adams M, et al. Gastrointestinal bleeding rates in recipients of nonpulsatile and pulsatile left ventricular assist devices. J Thorac Cardiovasc Surg. 2009;137:208-15.
3. Eckmann PM, John R. Bleeding and thrombosis in patients with continuous-flow ventricular assist devices. Circulation. 2012;125:3038-47.

4. Connors JM. Anticoagulation management of left ventricular devices. Am J Hematol. 2015;90:175-8.

5. Pollari F, Fischlein T, Fittkau M, Santarpino G. Anticoagulation with apixaban in left ventricular assist device patient with gastrointestinal bleeding: a viable alternative to warfarin? J Thorac Cardiovasc Surg. 2016;151: e79-81.

6. Guha A, Eshelbrenner CL, Richards DM, Monsur HP Jr. Gastrointestinal bleeding after continuous-flow left ventricular deice implantation: review of pathophysiology and management. Methodist Debakey Cardiovasc J. 2015;11: 24-7.

7. Suarez J, Patel CB, Felker GM, Becker R, Hernandez AF, Rogers JG. Mechanism of bleeding and approach to patients with axial flow left ventricular assist devices. Circ Heart Fail. 2011:4:779-84.

8. Rossi M, Serraino GF, Jiritano F, Renzulli A. What is the optimal anticoagulation in patients with a left ventricular assist device? Interact Cardiovasc Thorac Surg. 2012; 15:733-40.

9. Stulak JM, Lee D, Haft JW, Romano MA, Cowger JA, Park SJ, et al. Gastrointestinal bleeding and subsequent risk of thromboembolic events during support with a left ventricular assist device. J Heart Lung Transplant. 2014;33:60-4.

10. Heidbuchel H, Verhamme P, Alings M, Antz M, Diener HC, Hacke W, et al. Updated European Heart Rhythm Association practical guide on the use of non-vitamin $\mathrm{K}$ antagonist anticoagulants in patients with non-valvular atrial fibrillation. Europace. 2015;17:1467-507.

11. Miller CS, Grandi SM, Shimony A, Filion KB, Eisenberg MJ. Meta-analysis of efficacy and safety of new oral anticoagulants (dabigatran, rivaroxaban, apixaban) versus warfarin in patients with atrial fibrillation. Am J Cardiol. 2012; 110:453-60.

12. Eikelboom JW, Connolly SJ, Brueckmann M, Granger CB, Kappetein AP, Mack MJ, et al; RE-ALIGN Investigators. Dabigatran versus warfarin in patients with mechanical heart valves. $N$ Engl J Med. 2009;369:1206-14.

13. Hylek EM, Held C, Alexander JH, Lopes RD, De Caterina R, Wojdyla DM, et al. Major bleeding in patients with atrial fibrillation receiving apixaban or warfarin: The ARISTOTLE Trial (Apixaban for Reduction in Stroke and Other Thromboembolic Events in Atrial Fibrillation): predictors, characteristics, and clinical outcomes. J Am Coll Cardiol. 2014;63:2141-7. 